\title{
Nutzer, Sammler, Entscheidungsträger? Arten der Bürgerbeteiligung in Smart Cities
}

\author{
Jasmin Haunschild (D) - Kilian Demuth • Henri-Jacques Geiß • \\ Christian Richter $\cdot$ Christian Reuter iD
}

Eingegangen: 6. April 2021 / Angenommen: 20. Juli 2021 / Online publiziert: 17. August 2021

(C) Der/die Autor(en) 2021

Zusammenfassung Digitalisierung ist ein präsenter Faktor in vielen Städten. So existieren bereits viele Smart-City-Initiativen, bei denen Städte versuchen, ihre Prozesse durch Erfassung und Verknüpfung von Daten, oft unter Zuhilfenahme von Datenplattformen, zu optimieren. In Anbetracht der damit einhergehenden großen Investitionen und Veränderungen wird Bürgerbeteiligung als zentraler Faktor für den Erfolg solcher Initiativen betrachtet. Bisher ist allerdings nicht klar, was typische Beteiligungsformate von Smart-City-Initiativen sind und welche Rolle(n) BürgerInnen dabei einnehmen. Dieser Beitrag leitet mittels einer Literaturanalyse zu Smart Cities ein Kategorienschema zu typischen Bürgerbeteiligungsarten ab. Die Analyse ergab, dass sich Einbindung von BürgerInnen in politische Entscheidungen und bei der Entwicklung technischer Artefakte maßgeblich auf e-Government oder Participatory Design bezieht. Im Hinblick auf die Beteiligungsarten zeigt sich, dass Makrofabriken, Living Labs und Open-Data-Plattformen häufige Ansätze sind, um BürgerInnen als Co-Creators einzubinden. Zudem werden BürgerInnen mit Citizen Sensing zur Erfassung von Daten oder Missständen einbezogen. Dabei zeigen sich sowohl aktivere, als auch eher passive Beteiligungsarten. Die Analyse zeigt, dass die Einbindung von BürgerInnen häufig entweder auf eine Beteiligung an politischen Entscheidungen oder an der Entwicklung technischer Artefakte abzielt. Auch wenn keine klare Abgrenzung möglich ist, sind diese Ansätze dann eher durch e-Government oder Participatory Design inspiriert.

Schlüsselwörter Smart Cities · Bürgerbeteiligung · Co-Design · Co-Kreation · E-Government

\footnotetext{
Jasmin Haunschild $(\bowtie) \cdot$ Kilian Demuth $\cdot$ Henri-Jacques Geiß $\cdot$ Christian Richter $\cdot$ Christian Reuter Fachbereich Informatik, Lehrstuhl Wissenschaft und Technik für Frieden und Sicherheit (PEASEC), Technische Universität Darmstadt, Pankratiusstraße 2, 64289 Darmstadt, Deutschland E-Mail: haunschild@peasec.tu-darmstadt.de
} 


\title{
Users, Collectors, Decision Makers? Types of Participation Modes in Smart Cities
}

\begin{abstract}
Digitalization is currently a prominent factor in many cities. Already, many smart city initiatives exist that thrive to optimize processes by gathering and connecting data, often using data platforms. Considering the enormous financial investments and great changes, citizen participation is seen as a major success factor for such initiatives. So far it is unclear, what typical participation modes are in this context and which roles citizens take on in each. Therefore, this contribution reviews literature on smart city initiatives to derive categories of typical participation modes. Regarding typical participation mode, we find that citizens are involved as co-creators in micro factories, living labs, and open data platforms. In addition, with citizen sensing they are involved as data collectors. Other participation modes are geared towards political participation and aim for either active or passive participation. The analysis shows that participation aims either for political participation or for participation in the technical development. Although no clear separation can be made, the efforts are thus inspired mainly by e-government or participatory design.
\end{abstract}

Keywords Smart Cities · Citizen Participation · Co-Design · Co-Creation · E-Government

\section{Einleitung}

Die fortschreitende Digitalisierung mit der damit einhergehenden allumfassenden und dauerhaften Internetanbindung stellt ein großes Potenzial für die Entwicklung von neuen Technologien und Dienstleistungen zur Bereicherung und Vereinfachung des Alltags dar. Neben Smartphones sind mittlerweile auch Systeme in den Bereichen Smart Home sowie Smart Wearables weit verbreitet. Dies betrifft auch das urbane Leben: Unter dem Begriff Smart City begreifen sich bereits viele Städte als Vorreiter der Digitalisierung und erhoffen sich dadurch nicht nur Effizienzsteigerungen, sondern ebenfalls eine erhöhte Attraktivität des Standortes. Dabei wird angestrebt, mit Hilfe der Vernetzung intelligenter Systeme innerhalb einer Stadt oder einer Region verschiedene Lebensbereiche wie Arbeit, Logistik, Verkehr oder Verwaltung für die BewohnerInnen - trotz wachsender Bevölkerung - zu verbessern. Ein Kernaspekt für die smarte Gestaltung der Prozesse ist dabei typischerweise die Erfassung von Echtzeitdaten und ihre Verfügbarkeit durch Technologien wie Internet of Things (IoT), Wearable Devices und Cloud Computing (Ismagilova et al. 2019) und Datenerfassung durch private sowie öffentlicher Geräte wie Kameras und Sensoren.

Dabei ist das Konzept Smart City nicht unumstritten. Einerseits wird neben der Effizienzsteigerung auch das kreative Potenzial der BürgerInnen zur Innovation und bottom-up-Lösung urbaner Probleme durch die Zurverfügungstellung der Daten über Datenplattformen betont (Capdevila und Zarlenga 2015). Andererseits wurde auch gezeigt, dass das Konzept Smart City im Ursprung von wirtschaftlichen Interessen vorangetrieben wurde (Wiig 2016) und der Fokus auf BürgerInnen oft eine leere 
Sprachhülse bleibt (Sadowski und Bendor 2019). Arnsteins (1969) in der Wertigkeit aufsteigende, achtstufige Leiter der BürgerInnenbeteiligung zeigt, dass es bereits bei analoger Beteiligung große Unterschiede gibt im Hinblick auf die politische Relevanz der Mitwirkung. Während einige Arten dort als Nichtpartizipation oder Alibimaßnahme beschrieben werden, werden nur Partnerschaft, Machtdelegation und BürgerInnenkontrolle als Ansätze genannt, die einen Grad an Macht an BürgerInnen abgeben. Dabei kann Partizipation an allen Schritten im Politikzyklus stattfinden, von der Diskussion bestimmter Themen (agenda setting), über die Analyse, die Politikausrichtung (policy creation) bis zur Implementation und dem Monitoring. Die zur Partizipation genutzten Technologien sind dabei nur eine von zehn Dimensionen der Beteiligung, neben Aspekten wie involvierte Akteure, Zugang oder Ressourcen (Macintosh 2004). Dies verdeutlicht die Komplexität von Beteiligung und zeigt auch, dass der Einsatz von Technologie, abhängig von den anderen Dimensionen, dem Schritt im Politikzyklus und der Ambition der Machtabgabe an BürgerInnen, sehr unterschiedliche Effekte haben kann und nicht per se eine bedeutungsvollere Beteiligung darstellt.

Trotz eines großen Forschungsinteresses an Smart Cities (z.B. (Zanella et al. 2014), (Ismagilova et al. 2019)), an Aspekten der BürgerInnenbeteiligung und e-Government (z. B. (Degbelo et al. 2016), (Meijer und Bolívar 2016), (Viale Pereira et al. 2017)), auch anhand vergleichender Fallanalysen von Vorreiterstädten (z.B. (Okai et al. 2019), (Cardullo und Kitchin 2019), (Angelidou 2016)), fehlt bisher allerdings eine Systematisierung der typischen Arten der BürgerInnenbeteiligung in Smart Cities und deren politischen Beitrags.

Diese Arbeit untersucht daher anhand einer semistrukturierten Literaturanalyse, welche Arten der Partizipation in Smart-City-Projekten typischerweise existieren und welche Arten politischer Teilhabe dabei jeweils ermöglicht werden. Die Beantwortung dieser Fragen bietet einen Überblick über Trends aus der Information-SystemsPerspektive. Während dies keine Schlussfolgerungen über die Güte der Beteiligung zulässt, kann sich damit zeigen, welche Arten der Beteiligung in Smart Cities typischerweise technisch unterstützt und gefördert werden.

In Kapitel 2 wird dazu zunächst der Forschungsstand zur Rolle der BürgerInnen in Smart Cities analysiert und die Forschungslücke anhand der Forschungsrichtungen des Smart-City-Diskurses dargestellt. Daraufhin wird in Kapitel 3 die Methodik der Auswahl und Analyse der Literatur vorgestellt. Kapitel 4 präsentiert die Ergebnisse, bevor diese in Kapitel 5 ausgewertet und abschließend im Hinblick auf die Implikationen für Teilhabe an städtischer Digitalisierung diskutiert und resümiert werden.

\section{Bürgerbeteiligung in Smart Cities und Forschungslücke}

Es existieren viele Definitionen für Smart City und verwandte Beschreibungen wie zum Beispiel digital, intelligent oder ubiquitous city (Albino et al. 2015). Seit 2012 hat sich der Fokus innerhalb der Wirtschaftsinformatik beziehungsweise der Information Systems (IS) Literatur von einem technikzentrierten hin zu einem holistischeren Bild gewandelt, der sowohl verschiedene Dimensionen und menschliche Aspekte 
aufgreift (Ismagilova et al. 2019). Eine Definition, die die Aspekte aus IS-Perspektive verbindet, lautet: ,Smart cities use an IS centric approach to the intelligent use of ICT within an interactive infrastructure to provide advanced and innovative services to its citizens, impacting quality of life and sustainable management of natural resources" (Ismagilova et al. 2019, S. 90).

Smart-City-Initiativen sind bereits sehr verbreitet, unter anderem in allen EUMitgliedstaaten (Manville et al. 2014). Sie können viele Lebensbereiche wie Infrastruktur, Bildung, Lebensqualität und Regierung integrieren (z. B. in Stockholm und Barcelona), sich auf wenige Bereiche wie Innovation und Wirtschaft fokussieren (z.B. PlanIT Valley, Singapore) oder nur auf einen spezifischen Bereich, zum Beispiel Notfallprävention oder Wirtschaft (z. B. Rio, Konza) (Angelidou 2017). Nicht nur Metropolen, auch viele mittelgroße Städte verfolgen Smart-City-Ansätze (Bitkom 2019), die zum Teil auch gefördert werden, zum Beispiel 2017 durch von der Stadt Darmstadt gewonnen Wettbewerb „Digitale Stadt“ des Digitalverbands Bitkom in Zusammenarbeit mit dem Deutschen Städte- und Gemeindebund. Smart Cities werden zum Teil auch danach unterschieden, in welchem Ausmaß Technologie oder Menschen der treibende Faktor und Erfolgsmaßstab sind (Kummitha und Crutzen 2017) oder Menschen, Technologie oder Institutionen ,smarter“ werden (Nam und Pardo 2011). Zudem wird inhaltlich differenziert zwischen den Umweltfaktoren Smart Environment, Smart Mobility und Smart Economy und den eher sozialen Ebenen Smart Living, Smart People und Smart Governance (Giffinger et al. 2007), die auch für Rankings herangezogen werden (Cohen 2014). Smart Living beinhaltet dabei Aspekte wie Sicherheit, Wohnen oder Tourismus; Smart People Aspekte wie Diversität, Kreativität und Bildung; Smart Governance Aspekte wie öffentliche Dienstleistungen, Transparenz und Partizipation an politischen Entscheidungen (Giffinger et al. 2007).

Konzeptualisierung und Rechtfertigung von Smart Cities variieren laut Meijer und Bolívar (2016) in drei Dimensionen: Einerseits dahingehend, ob smarte Technologien, smarte Menschen oder smarte Kollaboration als die definierenden Faktoren betrachtet werden; ob von einem transformativen oder inkrementellem Wandel städtischer Governance, sowie ob als legitimierendes Ziel die Verbesserung der Ergebnisse oder ein offenerer Prozess angestrebt werden. Als weitere Vorteile werden oft die Einsparung von Ressourcen sowie Konkurrenzfähigkeit der Smart Cities als Standort der Innovation genannt (Angelidou 2015).

Dabei sind aber auch Kosten und Risiken abzuwägen. Neben hohen Kosten für Systemintegration und Wartung kann die verbreitete Verfügbarkeit und Nutzung von Informations- und Kommunikationstechnologie (IKT) auch durch große Angriffsflächen zu gravierenden Ausfällen und Datenlecks führen (Reuter et al. 2020). Zudem werden sozio-technische Herausforderungen, besonders bei den Themen Privatheit und Überwachung (Angelidou 2016), sowie bei Teilhabe und Inklusion (Lawton et al. 2014) gesehen. Besonders im öffentlichen Raum und in Interaktion mit dem öffentlichen Sektor, wo hierarchische Entscheidungen gefällt werden und BürgerInnen der Implementation neuer Technologien stärker ausgesetzt sind, sind Aspekte der Teilhabe und der Technologieakzeptanz besonders virulent (Kitchin 2015).

In Reaktion auf Kritiken wird zunehmend ein bürgerInnenzentrierter Ansatz im Sinne einer Human Smart City gefordert und beworben (Oliveira und Campolargo 
2015; Cardullo und Kitchin 2019). Dabei sollen einerseits die Vorteile für BürgerInnen in den Fokus gestellt werden, andererseits die Vergrößerung der Relevanz für BürgerInnen durch Beteiligung an Entscheidungen und technischen Entwicklungen gefördert werden. Äquivalent dazu definiert und legitimiert sich Smart Governance durch mit smarten Diensten verbesserte Ergebnisse (z. B. im Hinblick auf Wohlstand oder Gesundheit) oder verbesserte Prozesse (z.B. offener Teilhabe und Beteiligung) (Meijer und Bolívar 2016).

Wichtige Voraussetzungen für die Beteiligung in Smart-City-Initiativen sind die Informiertheit, ausreichende Kapazitäten und die Motivation der BürgerInnen als weiterer Stakeholder in den Prozess der Smart-City-Entwicklung teilzunehmen. Diese Themen werden in aktueller Forschung kaum adressiert (Simonofski et al. 2019). Es gibt jedoch Hinweise darauf, dass politische Wirksamkeit, das Zugehörigkeitsgefühl zur Stadt, sowie die Zufriedenheit mit der öffentlichen Verwaltung Motivatoren sind, die die Beteiligung von BürgerInnen begünstigen (Lebrument et al. 2021). Zudem gibt es Ansätze das Engagement von BürgerInnen zu fördern, indem man die Plattformen, die oftmals von Smart-City-Initiativen für die BürgerInnen zur Verfügung gestellt werden, bereits an die Bedürfnisse der NutzerInnen anpasst (Simonofski et al. 2021). Des Weiteren zeigt aktuelle Forschung, dass Bürgerbeteiligung zu mehr Zufriedenheit in Smart Cities führen kann (Xu und Zhu 2020).

Zur Analyse von Beteiligung beschreibt Macintosh (2004) die drei qualitativ aufbauenden Ebenen enabling (nutzerfreundliche Bereitstellung von Informationen), engaging (top-down Konsultation und Feedback) und empowering (aktive Beteiligung und Förderung von bottom-up Ideen). In ähnlicher Art beschreibt das OECDFramework „Citizens as Partners“ (2001) die drei Schritte Information, Konsultation und aktive Partizipation. Im Hinblick auf die Nutzung von Technologie zeigt Design Science Research dabei, dass die AnwenderInnen in den verschiedenen Phasen des Prozesses eingebunden sein können. Typischerweise erfolgt die Einbindung auf der Ebene der Co-Implementation bei der Einführung einer Technologie, zum Teil auch als Co-Design im Design-Prozess, selten als Co-Initiation bei der Auswahl (Sørensen und Torfing 2018).

Welche typischen Beteiligungsformen sich bisher in Smart Cities herausgebildet haben und wie sie sich an den Schnittstellen zwischen Smart Cities, Information Systems Design und e-Government einordnen, stellt bisher eine Forschungslücke dar. Im Folgenden wird daher eine Kategorisierung von Beteiligungsarten in Smart Cities vorgenommen und Gemeinsamkeiten und Unterschiede analysiert. Die Arbeit eröffnet somit einen Überblick über typische Beteiligungsformen und einige Anwendungsbeispiele und bietet eine Einordnung der Beteiligungstypen zwischen partizipativem Design und politischer Beteiligung.

\section{Methodisches Vorgehen}

Um einen Überblick über die typischen Arten der Beteiligung von BürgerInnen in Smart Cities zu gewinnen, wird im Folgenden eine Literaturanalyse durchgeführt. Diese beinhaltet zunächst eine Suche nach relevanten wissenschaftlichen Arbeiten, deren Inhalt systematisch extrahiert und synthetisiert wird (Weed 2005). Auswahl 
und Analyse der Artikel orientieren sich an der Leitfrage: Auf welche Arten werden BürgerInnen in Smart-City-Ansätzen typischerweise beteiligt? Eine initiale Recherche zu der Leitfrage liefert die englischen Schlüsselbegriffe Governance, Participation, Social Innovation, Engagement, Collaboration und Co-Creation. Diese werden in Kombination mit „Smart Cit*“ in den Datenbanken Web of Science und Google Scholar gesucht. Um die bedeutendsten Beiträge zu identifizieren wurden im Web of Science alle Beiträge mit mehr als 50 Zitationen berücksichtigt. Um dabei neuere Beiträge, die noch weniger Zitate haben, nicht zu übersehen, wurden zudem die thematisch relevantesten Beiträge gefiltert und hier besonders jene berücksichtigt, die Anwendungsfälle analysieren. Zudem wurden die zu jeder Kombination der Schlüsselbegriffe gefundenen 20 ersten Beiträge von Google Scholar genutzt, welches das Auftretens der Begriffe im gesamten Text, der Anzahl an Zitationen insgesamt und in letzter Zeit berücksichtigt und sowohl in Wissenschaft als auch Praxis häufig genutzt wird (https://scholar.google.com/scholar/about.html, Brown 2017). Duplikate und nicht-wissenschaftliche Texte wurden aussortiert.

Die resultierenden Quellen wurden zunächst anhand von Titel und Abstract, im nächsten Schritt anhand des Textes nach Relevanz bezüglich der Leitfrage gefiltert. Die finalen 49 Beiträge wurden anhand eines induktiv entstehenden Kategorieschemas klassifiziert. Die Auswahl der Texte ist nicht repräsentativ oder alle möglichen Aspekte umfassend. Stattdessen stellt sie die typischen Erscheinungsarten von Bürgerbeteiligung speziell im Kontext von Smart-City-Initiativen dar.

\section{Ergebnisse}

Im Folgenden werden anhand einer Kategorisierung der Literatur drei Arten der Bürgerbeteiligung identifiziert und erläutert: zuerst die Beteiligung als Co-Creator, in der BürgerInnen als Innovatoren fungieren; anschließend die Beteiligung durch Citizen-Sensing, wobei BürgerInnen die Rolle der Datensammler annehmen; und abschließend die politische Beteiligung von sogenannten Smart Citizens.

\subsection{Beteiligung als Co-Creator - BürgerInnen als Innovatoren}

Eine Rolle, die Smart Cities eröffnen können, ist die der Co-Creators, wobei BürgerInnen zum kollaborativen Mitwirken an Innovationen ermächtigt und befähigt werden. Zentral sind dabei Orte in der Stadt, die eine solche Zusammenarbeit ermöglichen. Diese „Mikrofabriken“, in der Literatur alternativ als Makerspaces, Hackerspaces, Fab-oder Media-Labore bezeichnet, bieten ,Aktivisten, Hackern, Wissenschaftlern und Anderen“ (Niaros 2016, S. 56; Übersetzung der Autoren) Platz und oft auch besonders technische Ausstattung um Probleme des Stadtalltags zu lösen und ihren Lebensraum zu gestalten. Instanzen solcher Mikrofabriken sind beispielsweise das Metalab in Wien, das Medialab-Prado in Madrid oder das ,design collective: mobility" in München (Farías und Widmer 2017, S. 53; Meijer et al. 2019). Ein weiteres Beispiel ist das Air Quality Egg, das Freiwillige aus Amsterdam, London, New York und Barcelona in Zusammenarbeit als ,kleinen Computer mit Sensoren zum Messen von Substanzen in der Luft“" (Zandbergen 2017, S. 541) erarbeiten. Eben- 
falls ein häufig vertretenes Vorgehen bei der Umsetzung von Co-Creation ist das Benutzen der Stadt oder bestimmter Distrikte als lebendes Labor (Living Laboratory). Diese Living Labs sind bestimmte Bereiche einer Stadt, die EntwicklerInnen und EntrepreneurInnen die Möglichkeit geben, neue Produkte und Ideen innerhalb der „Dynamiken des alltäglichen Lebens“ (Spagnoli et al. 2019, S. 139; Übersetzung der Autoren) zu testen, und oft den BürgerInnen einen direkten Weg für Feedback, Kritik und Ideen zu den getesteten Produkten und Services bieten. Die BürgerInnen können hierbei bei allen drei Phasen der Living Labs - Design, Implementierung und Evaluation - zentral in den Prozess eingebunden werden. Beispielsweise wurden BürgerInnen im Projekt Nexthamburg über ein Jahr lang durch ,Workshops, Online Dialog und einen Ideenwettbewerb“ (Menny et al. 2018, S. 72; Übersetzung der Autoren) in den Entwurf eines Living Labs einbezogen. Das erste Ziel des Projekts 2008 war es den BürgerInnen einen neuen Raum für die Beteiligung an der Stadtplanung zu bieten. Dafür wurde eine Online-Plattform generiert, auf der jegliche Ideen von BürgerInnen ihren Platz und Wertschätzung erhielten. Die Ideen wurde mittels verschiedener Methoden (z. B. Online-Dialog, Sessions, Zukunftscamp) zu einer Bürgervision zusammengefasst, welche ein Zukunftsszenario von Hamburg im Jahr 2030 darstellt (Petrin 2012).

Eine weitere und bereits oft umgesetzte Strategie, um BürgerInnen zu EntrepreneurInnen und Co-Creators werden zu lassen, ist die sogenannte Open-Data Policy, basierend auf den weitläufig in der Stadt erhobenen Daten. Open Data sind maschinenlesbare Daten, die der Öffentlichkeit zur Verfügung gestellt werden (Strömberg und Näslund 2017), weshalb personenbezogene Daten und solche, die Rückschlüsse auf Personen zulassen, nicht enthalten sein sollten. Häufig werden dazu Datenplattformen etabliert, wie die Open Source-Plattformen FIWARE (Araujo et al. 2019) und CityPulse (Giatsoglou et al. 2016) oder proprietäre Angebote wie Microsoft.CityNext (Wilson et al. 2013), die die Zusammenführung verschiedener Datentypen ermöglichen. Die zur Verfügung gestellten Daten können von Verwaltungen, Unternehmen oder Privatpersonen generiert sein und alle oder Teile der in der Stadt generierten Daten beinhalten, inklusive im Smart-City-Kontext installierter Sensoren. Gerade in Kombination mit Mikrofabriken bieten Open Data die Möglichkeit, selbst Anwendungen zu entwickeln, wobei auch Open-SourceAnwendungen und Schnittstellen (engl. application programming interfaces, APIs) zur einfacheren Nutzung der Datenbanken angeboten werden können, wie beispielsweise in Häme in Finnland für Event-Informationen (Jussila et al. 2019). Die Daten können ebenfalls genutzt werden, um die Regierung auf Missstände hinzuweisen oder Aufmerksamkeit für diese zu schaffen (Capdevila und Zarlenga 2015) und sie können für mehr Transparenz bei Entscheidungen sorgen (Cardullo und Kitchin 2019). Hivon und Titah (2017) zeigen, dass auch bei der Entwicklung von Open-Data-Plattformen NutzerInnen involviert werden sollten, sodass diese problemorientiert und für BürgerInnen nützlich wird.

Zusammenführend können also Mikrofabriken im Zusammenspiel mit einer Open-Data-Policy und der Nutzung der Stadt als Living Laboratory dazu genutzt werden, die Smart Cities in Zusammenarbeit mit und durch Zusammenarbeit unter den Bürgern zu entwickeln und zu implementieren. Dieser Ansatz wird in der 
Literatur oft als bottom-up bezeichnet und als ideal für innovative Smart Cities im Allgemeinen angesehen (Angelidou 2017).

\subsection{Beteiligung durch Citizen Sensing - BürgerInnen als Datensammler}

Ein Charakteristikum städtischer Digitalisierung ist das Verknüpfen von Sensordaten und IKT, um darauf basierend Prozesse zu optimieren und in Echtzeit auf Ereignisse reagieren zu können. Hierbei können auch BürgerInnen als DatensammlerInnen und somit als Teil des IKT-Netzwerks beteiligt sein. Im Allgemeinen lässt sich dieses Konzept der BürgerInnen als „Sensoren, die rechnergestützte Technologien verwenden, um Feedback zu geben“ (Billert und Peters 2019, S. 172) als Citizen Sensing bezeichnen. Dies geschieht beispielsweise mit der Smartphone-Anwendung Waze (Waze 2020), bei der Reisedaten von NutzerInnen aufgezeichnet und analysiert werden, inklusive von Nutzenden erhobener Medien wie Bilder von Verkehrsunfällen. Auf diese Art generierte Datensätze werden beispielsweise in Rio de Janeiro dazu genutzt, Machine-Learning-Anwendungen zu trainieren und Probleme im Stadtverkehr aufzuzeigen (Viale Pereira et al. 2017). Neben diesen Echtzeitdaten gibt es auch Instanzen dieses Citizen Sensing mit längerfristiger Nutzung der Daten. Ein Beispiel hierfür ist NYC311, eine Anwendung für New Yorker mit Informationen über und Kontaktvermittlung zu Regierungsservices von Obdachlosenhilfe zu Kinderbetreuung, aber auch zum Melden von Beschwerden oder Missständen, zum Beispiel von defekten Laternenpfählen (Mainka et al. 2016). Ein weiteres Beispiel ist das in der Mikrofabrik Fab Lab Barcelona entwickelte Smart Citizen Kit. Hierbei handelt es sich um ein Open-Source Soft- und Hardware-Projekt zum Aufzeichnen von Parametern der Umwelt und Luft (Capdevila und Zarlenga 2015). Die benötigte Soft- und Hardware wird von kollaborierenden BürgerInnen in einer Mikrofabrik entwickelt und wiederum von BürgerInnen zum Aufzeichnen von Umweltdaten genutzt, die dann in den Mikrofabriken wieder für das Entwickeln neuer Anwendungen und Lösungen genutzt werden können. Die hier beschriebenen Beispielprojekte sammeln mit der Hilfe von NutzerInnen reale Daten über Verkehr, kommunale Problemstellungen wie Straßenschäden, fehlende Beleuchtung oder ökologische Aspekte, wie den Stand der Luftverschmutzung, die andernfalls den administrativen EntscheidungsträgerInnen oder EntrepreneurInnen nicht zur Verfügung stünden. Ebenfalls zeigt sich ein Synergiepotential zwischen Citizen Sensing und Mikrofabriken. Shelton et al. (2015) zeigen am Beispiel Louisville aber auch, dass BürgerInnen auch politische Narrative kontestierende Daten erheben können. Hier hat eine Bürgerbewegung durch analoge BürgerInnenbefragungen und Datenerhebung vor Ort die staatlichen Zahlen zum Leerstand von Gebäuden widerlegt und ein größeres Ausmaß des Problems aufgezeigt.

\subsection{Politische Beteiligung von Smart Citizens}

Eine weitere zentrale Perspektive sieht BürgerInnen als ExpertInnen ihrer Nachbarschaft, die spezielles lokales Wissen besitzen. Dieses Wissen sollt in der Stadtplanung und durch die Nutzung von IKT verstärkt in die Entscheidungsfindung und Lokalpolitik der Smart Cities eingebunden werden. Die IKT-gestützte Beteiligung 
und der verstärkte Austausch zwischen Behörden und Politik (Mellouli et al. 2014) wird auch mit den Begriffen e-Governance, e-Democracy oder Smart Governance beschrieben.

Dabei lässt sich zwischen passiver und aktiver Beteiligung unterscheiden. Aktive Beteiligung liegt vor, wenn BürgerInnen direkt mit der Politik und den Behörden interagieren oder für politisch-administrative Entscheidungen herangezogen werden häufig unter Nutzung von IKT. So konnten BürgerInnen in Gent Ideen für SmartCity-Innovationen einreichen und über ihre Aufnahme zur Wahl, sowie die Auswahl der Ideen selbst entscheiden (Schuurman et al. 2012). Dabei wurde festgestellt, dass die Ideen zwar nicht sehr innovativ, allerdings von großem praktischem Nutzen für die BürgerInnen waren. So wird beispielsweise unmittelbar bei der Konstruktion von neuen Gebäuden und Distrikten das Wissen der BewohnerInnen als „Experten ihres Alltags“ (Billert und Peters 2019, S. 172) miteinbezogen. Ein weiteres Beispiel ist crowdsourcing. Statt ExpertInnen zu befragen, wird bei Crowdsourcing das Wissen oder Beobachtungen der „Menge“, also der breiten BürgerInnenschaft angefragt. Dabei wird die Mithilfe der BürgerInnen aktiv angefordert, um lokale Informationen zu erhalten oder Einschätzungen abzugeben (Benouaret et al. 2013).

Unter passive Beteiligung fallen Konzepte, bei denen sich BürgerInnen beispielsweise über Social Media untereinander austauschen und sich dadurch ein Dialog und gegebenenfalls Konsens bildet, welcher die Politik und die Behörden beeinflusst (Alawadhi et al. 2012; Gabrys 2014). In einer passiven Rolle kann zudem Citizen Sensing genutzt werden, um auf Grundlage der so erhobenen Daten politische Entscheidungen, zum Beispiel im Straßenbau zur Verbesserung der Verkehrsführung, zu treffen. BürgerInnen dienen in vielen Fällen primär als Informationsquelle, beispielsweise für Smartphone-Umfragen der Stadt Hamburg (Spil et al. 2017), oder für das Centro De Operacoes Prefeitura Do Rio, eine zentrale Datensammelstelle in Rio de Janeiro (Kitchin 2014). Verwaltung und Politik können aber auch durch IKT direkt Feedback und Meinungen zu Vorhaben und Projekten einholen (Alawadhi et al. 2012). Ein Beispiel ist ein Projekt in Namibia, wo BürgerInnen mittels Virtual Reality Pläne der Administration in Bezug auf Städte- und Wohnungsbau visuell vorgestellt und Feedback und Anregungen zur Verbesserung eingeholt wurden (Oksman und Raunio 2018). Für eine möglichst breite Einbindung können öffentliche Bildschirme und Terminals genutzt werden (Hosio et al. 2014).

\subsection{Kategorisierung der Bürgerbeteiligung in der Literatur}

Die folgende Tab. 1 zeigt die in der Analyse identifizierten typischen Beteiligungsarten. Bei Co-Creation wird zwischen den Ansätzen der Mikrofabrik, der Open-DataPolicy sowie den Living Labs, sowie im Fall von politischer Beteiligung zwischen aktiv und passiv unterschieden. Dabei zeigt sich kein klares Bild von zusammen oder separat auftretenden und adressierten Beteiligungsarten. Zum Teil wird BürgerInnen eine weitere Rolle als Stakeholder zugesprochen. Hierbei handelt es sich allerdings häufig um eine abstrakte Vision von BürgerInnen in Smart Communities, die in Technologien einen Mehrwert sehen und sich zu deren Gestaltung einbringen (Portmann und Finger 2015). Diese Vision hat allerdings keine konkrete Umsetzungsform und findet sich daher nicht in der Kategorisierung. 
Tab. 1 Arten der Bürgerbeteiligung, sortiert nach Citizen-Sensing

\begin{tabular}{|c|c|c|c|c|c|c|}
\hline \multirow{2}{*}{$\begin{array}{l}\text { Kategorie } \\
\text { Quelle und empirische Fälle }\end{array}$} & \multirow{2}{*}{$\begin{array}{l}\text { Citizen } \\
\text { Sensing }\end{array}$} & \multicolumn{3}{|c|}{ BürgerInnen als Co-Creators } & \multicolumn{2}{|c|}{ Pol. Beteiligung } \\
\hline & & $\begin{array}{l}\text { Mikro- } \\
\text { fabrik }\end{array}$ & $\begin{array}{l}\text { Open } \\
\text { Data }\end{array}$ & $\begin{array}{l}\text { Living } \\
\text { Labs }\end{array}$ & Aktiv & Passiv \\
\hline $\begin{array}{l}\text { Capdevila und Zarlenga (2015) } \\
\text { Barcelona }\end{array}$ & - & - & - & - & 0 & $\mathrm{O}$ \\
\hline $\begin{array}{l}\text { Angelidou (2017) } \\
\text { Amsterdam, Barcelona, Chicago, Cy- } \\
\text { berjaya, King Abdullah Economic City, } \\
\text { Konza, London, Masdar City, New } \\
\text { York, PlanITValley, Rio de Janeiro, } \\
\text { Singapur, Skolkovo, Stockholm, Song- } \\
\text { do }\end{array}$ & $\bullet$ & $\bullet$ & $\bullet$ & 0 & $\bullet$ & $\bullet$ \\
\hline $\begin{array}{l}\text { Farías und Widmer (2017) } \\
\text { München }\end{array}$ & $\bullet$ & $\bullet$ & $\bullet$ & O & $\bullet$ & $\bullet$ \\
\hline Zandbergen (2017) & $\bullet$ & $\bullet$ & $\bullet$ & O & O & O \\
\hline $\begin{array}{l}\text { Cardullo und Kitchin (2019) } \\
\text { Dublin }\end{array}$ & $\bullet$ & $\bullet$ & 0 & $\bullet$ & 0 & 0 \\
\hline Meijer et al. (2019) & $\bullet$ & $\bullet$ & $\bigcirc$ & O & $\bullet$ & $\bullet$ \\
\hline $\begin{array}{l}\text { Effing und Groot (2016) } \\
\text { Berlin, Krakau, Peking, Reykjavík, } \\
\text { Seoul }\end{array}$ & $\bullet$ & $\bullet$ & $\bigcirc$ & O & $\bullet$ & $\bullet$ \\
\hline $\begin{array}{l}\text { Spil et al. (2017) } \\
\text { Berlin, Eschede, Hamburg }\end{array}$ & $\bullet$ & $\bullet$ & 0 & 0 & $\bullet$ & $\bullet$ \\
\hline $\begin{array}{l}\text { Hosio et al. (2014) } \\
\text { Oulu }\end{array}$ & $\bullet$ & $\bullet$ & $\bigcirc$ & ○ & O & $\bigcirc$ \\
\hline Kitchin (2014) & $\bullet$ & $\bullet$ & $\bigcirc$ & ○ & $\bigcirc$ & O \\
\hline Simonofski et al. (2019) & $\bullet$ & $\bigcirc$ & $\bullet$ & $\bullet$ & $\bullet$ & $\bullet$ \\
\hline Castelnovo et al. (2016) & $\bullet$ & O & $\bullet$ & 0 & $\bullet$ & 0 \\
\hline $\begin{array}{l}\text { Mainka et al. (2016) } \\
\text { Aarhus, Helsinki }\end{array}$ & $\bullet$ & O & $\bigcirc$ & $\bullet$ & $\bullet$ & O \\
\hline $\begin{array}{l}\text { Viale Pereira et al. (2017) } \\
\text { Belo Horizonte, Porto Alegre, Rio de } \\
\text { Janeiro }\end{array}$ & $\bullet$ & O & $\bigcirc$ & $\bigcirc$ & $\bullet$ & $\bullet$ \\
\hline $\begin{array}{l}\text { Alawadhi et al. (2012) } \\
\text { Mexico City, Philadelphia, Quebec } \\
\text { City, Seattle }\end{array}$ & $\bullet$ & O & 0 & O & $\bullet$ & $\bullet$ \\
\hline Granier und Kudo (2016) & $\bullet$ & 0 & 0 & 0 & $\bullet$ & $\bullet$ \\
\hline Gabrys (2014) & $\bullet$ & ○ & $\bigcirc$ & $\bigcirc$ & ○ & $\bullet$ \\
\hline Benouaret et al. (2013) & $\bullet$ & 0 & 0 & 0 & 0 & $\bullet$ \\
\hline Billert und Peters (2019) & $\bullet$ & 0 & 0 & 0 & 0 & 0 \\
\hline $\begin{array}{l}\text { Bakici et al. (2013) } \\
\text { Barcelona }\end{array}$ & $\bullet$ & $\bigcirc$ & $\bigcirc$ & $\bigcirc$ & O & $\bigcirc$ \\
\hline Reforgiato Recupero et al. (2016) & $\bullet$ & $\bigcirc$ & $\bigcirc$ & $\bigcirc$ & $\bigcirc$ & $\bigcirc$ \\
\hline Benevolo et al. (2016) & $\bullet$ & O & $\bigcirc$ & ○ & $\bigcirc$ & O \\
\hline Vanolo (2014) & $\bullet$ & O & 0 & $\bigcirc$ & 0 & O \\
\hline Niaros (2016) & 0 & $\bullet$ & $\bullet$ & $\bullet$ & $\bullet$ & $\bullet$ \\
\hline $\begin{array}{l}\text { Angelidou (2016) } \\
\text { Amsterdam, Barcelona, London, Stock- } \\
\text { holm }\end{array}$ & 0 & $\bullet$ & $\bullet$ & $\bullet$ & O & O \\
\hline
\end{tabular}


Tab. 1 (Fortsetzung)

\begin{tabular}{|c|c|c|c|c|c|c|}
\hline \multirow{2}{*}{$\begin{array}{l}\text { Kategorie } \\
\text { Quelle und empirische Fälle }\end{array}$} & \multirow{2}{*}{$\begin{array}{l}\text { Citizen } \\
\text { Sensing }\end{array}$} & \multicolumn{3}{|c|}{ BürgerInnen als Co-Creators } & \multicolumn{2}{|c|}{ Pol. Beteiligung } \\
\hline & & $\begin{array}{l}\text { Mikro- } \\
\text { fabrik }\end{array}$ & $\begin{array}{l}\text { Open } \\
\text { Data }\end{array}$ & $\begin{array}{l}\text { Living } \\
\text { Labs }\end{array}$ & Aktiv & Passiv \\
\hline $\begin{array}{l}\text { Spagnoli et al. (2019) } \\
\text { Antwerpen, Carouge/Genf, Eindhoven, } \\
\text { Helsinki, Mailand, Manchester, Porto, } \\
\text { Santander }\end{array}$ & $\bigcirc$ & $\bullet$ & $\mathrm{O}$ & $\bullet$ & $\bullet$ & $\bigcirc$ \\
\hline Strömberg und Näslund (2017) & 0 & 0 & 0 & 0 & 0 & 0 \\
\hline Cardone et al. (2013) & $\bigcirc$ & 0 & $\bigcirc$ & 0 & $\bigcirc$ & $\bigcirc$ \\
\hline $\begin{array}{l}\text { Shelton et al. (2015) } \\
\text { Louisville, Philadelphia }\end{array}$ & $\bigcirc$ & $\bullet$ & $\bigcirc$ & $\bigcirc$ & $\bigcirc$ & $\bigcirc$ \\
\hline $\begin{array}{l}\text { Hu et al. (2013) } \\
\text { Vancouver }\end{array}$ & 0 & $\bullet$ & O & O & $\bigcirc$ & O \\
\hline $\begin{array}{l}\text { Komninos et al. (2013) } \\
\text { Barcelona, Helsinki, Manchester, Thes- } \\
\text { saloniki }\end{array}$ & $\bigcirc$ & O & $\bullet$ & $\bullet$ & $\bigcirc$ & 0 \\
\hline Bencardino und Greco (2014) & 0 & 0 & $\bullet$ & $\bigcirc$ & $\bullet$ & $\bullet$ \\
\hline Nam und Pardo (2011) & 0 & 0 & $\bullet$ & 0 & $\bullet$ & • \\
\hline $\begin{array}{l}\text { Oliveira und Campolargo (2015) } \\
\text { Aalborg, Birmingham, Lissabon, Mai- } \\
\text { land }\end{array}$ & 0 & O & $\bullet$ & $\bigcirc$ & $\bullet$ & O \\
\hline Lytras et al. (2019) & 0 & 0 & $\bullet$ & 0 & 0 & - \\
\hline Gooch et al. (2015) & ○ & 0 & $\bullet$ & $\bigcirc$ & O & $\bigcirc$ \\
\hline $\begin{array}{l}\text { Meijer und Thaens (2018) } \\
\text { Eindhoven }\end{array}$ & 0 & 0 & 0 & $\bullet$ & 0 & $\bullet$ \\
\hline $\begin{array}{l}\text { Chang (2019) } \\
\text { Amsterdam }\end{array}$ & 0 & 0 & 0 & $\bullet$ & 0 & 0 \\
\hline Menny et al. (2018) & 0 & 0 & 0 & $\bullet$ & 0 & 0 \\
\hline Degbelo et al. (2016) & ○ & 0 & 0 & $\bullet$ & O & $\bigcirc$ \\
\hline $\begin{array}{l}\text { Jussila et al. (2019) } \\
\text { Häme/Hämeenlinna }\end{array}$ & $\bigcirc$ & O & O & $\bullet$ & $\bigcirc$ & 0 \\
\hline $\begin{array}{l}\text { Hivon und Titah (2017) } \\
\text { Montréal }\end{array}$ & 0 & 0 & 0 & $\bullet$ & 0 & 0 \\
\hline $\begin{array}{l}\text { Boes et al. (2015) } \\
\text { Amsterdam, Barcelona, Helsinki }\end{array}$ & 0 & O & $\bigcirc$ & 0 & $\bullet$ & • \\
\hline $\begin{array}{l}\text { Oksman und Raunio (2018) } \\
\text { Keetmanshoop }\end{array}$ & ○ & O & O & 0 & $\bullet$ & $\bullet$ \\
\hline Pellicano et al. (2019) & 0 & 0 & $\bigcirc$ & $\bigcirc$ & $\bullet$ & $\bullet$ \\
\hline $\begin{array}{l}\text { Chatfield und Reddick (2016) } \\
\text { Kitakyushu }\end{array}$ & 0 & 0 & O & O & $\bullet$ & $\bullet$ \\
\hline Yeh (2017) & $\bigcirc$ & O & $\bigcirc$ & $\bigcirc$ & $\bullet$ & $\bullet$ \\
\hline Mellouli et al. (2014) & 0 & 0 & $\bigcirc$ & $\bigcirc$ & • & $\bullet$ \\
\hline Cantador et al. (2020) & 0 & 0 & $\bigcirc$ & 0 & $\bullet$ & 0 \\
\hline
\end{tabular}




\section{Diskussion}

Die Analyse zeigt, dass sich verschiedene spezifische Arten der Bürgerbeteiligung im Kontext von Smart Cities etabliert haben, die sich häufig durch den Einsatz neuer Technologien und verknüpfter Daten von konventionellen Arten der Beteiligung - die häufig auf informieren und One-Way-Kommunikation beschränkt sind - unterscheiden. Bei der Beteiligung der BürgerInnen innerhalb von Smart Cities hat sich als eine ambitionierte Rolle die der Co-Creatoren gezeigt, wobei BewohnerInnen durch zu diesem Zwecke gestaltete Räume (Mikrofabriken), durch die Bereitstellung von Daten (Open Data) oder durch Testumgebungen (Living Labs) selbst an Problemen arbeiten und technische Lösungen entwickeln können. Eine weitere Rolle für BürgerInnen ist das Agieren als Sensor beziehungsweise Datensammler (Citizen Sensing) im IKT-Netzwerk der Stadt. Dies ist beispielsweise über Smartphone-Anwendungen möglich, wobei NutzerInnen passiv zum Beispiel durch das Aufzeichnen von durch das Smartphone gemessenen Daten (z. B. Reisedaten) oder aktiv durch das Fotografieren und Melden von zum Beispiel Verkehrsunfällen als Sensor der Stadt agiert. Durch dieses Citizen Sensing und den freiwilligen Beitrag von oftmals geo-referenzierten Informationen (Goodchild 2007) können Synergieeffekt mit Open Data auftreten. Eine andere Art der Beteiligung kann in politischer Entscheidungsfindung bestehen. Dies beinhaltet zum einen die passive Partizipation der BürgerInnen durch das Wahrnehmen und Miteinbeziehen der öffentlichen Meinung über digitale Kanäle wie zum Beispiel soziale Netzwerke. Zudem werden häufig eigene Kanäle geschaffen, über die BürgerInnen lokales Wissen über Störungen oder Missstände melden oder Feedback und Anregungen geben können.

Dabei ist zu unterscheiden zwischen einem Melden von Beobachtungen, das eher als Citizen Sensing zu verstehen ist, und einer Involvierung in politische Debatten und Entscheidungen über die Priorisierung von Herausforderungen des Stadtlebens. In beiden Fällen - wie auch bei der Entwicklung technischer Artefakte - sind BürgerInnen als „Experten ihres Alltags“ (Billert und Peters 2019, S. 172) zu verstehen, die den administrativen Organen ihr lokales Wissen zur Problemidentifikation oder zur Entscheidungsfindung zur Verfügung stellen. Im Smart-City Kontext werden dafür häufig Online-Plattformen eingerichtet. Technik wirkt dabei nicht notwendigerweise transformativ, da auch neue Technologien wie Soziale Medien genutzt werden können, um auf der Ebene des Informierens zu verharren. Abb. 1 zeigt eine Zuordnung

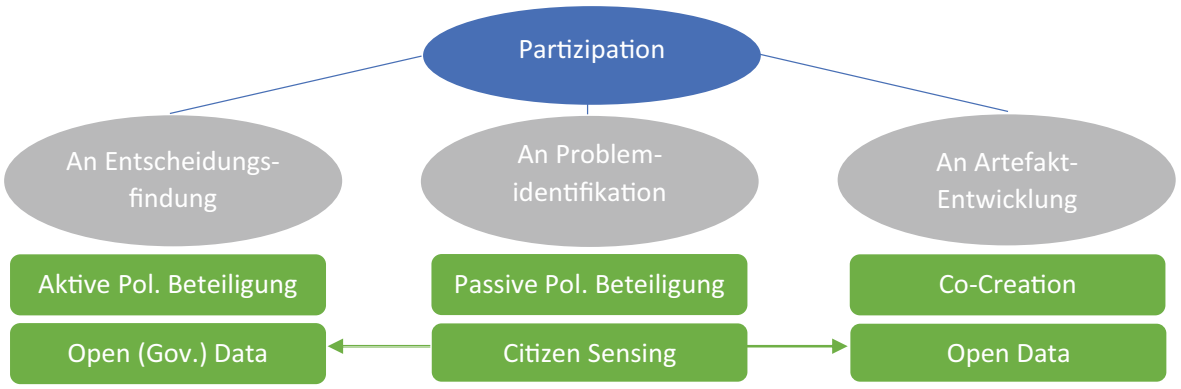

Abb. 1 Beiträge der Partizipationsformen in Smart Cities 
der typischen Beteiligungsarten in Smart Cities zu der politischen Dimension der Entscheidungsfindung (durch Open Government Data und aktive Beteiligung) und zur Entwicklung technischer Artefakte (durch Co-Creation und durch Open Data).

Dabei zeigt sich, dass es zwischen diesen beiden aktiven Beteiligungsebenen noch eine dritte gibt, bei der Daten eher technokratisch genutzt werden und BürgerInnen nur passiv zum Beispiel durch Abstimmungen oder durch Meldungen von Missständen eingebunden sind. Solche Meldungen können wiederum in Open-DataPlattformen einfließen und als Indikator für das Vorliegen von Problemen genutzt werden und damit als Grundlage für die Entwicklung von Anwendungen oder als Grundlage für politische Entscheidungen dienen. Eine einfache Aussage anhand der genutzten Artefakte ist also weder über das Ausmaß der politischen Einbindung noch über den Beitrag zur technischen oder politischen Gestaltung zu treffen, da die Artefakte Wechselwirkungen aufzeigen und zu beiden Ebenen beitragen können.

Die Analyse suggeriert, dass Aspekte der kollaborativen Innovation, besonders Co-Creation, die mitunter auch als Reaktion auf die Kritik der Smart Cities als vom Kapitalismus getragenes Projekt zu verstehen ist, häufig zu finden sind in SmartCity-Projekten, indem hier dafür designierte Orte geschaffen werden. Eine ähnlich zentrale Verankerung gibt es für Smart Governance in Form von Online-Plattformen, allerdings gibt es keinen designierten physischen Ort für deren Gestaltung. Andererseits laufen Co-Creation-Projekte auch Gefahr, als Alibipolitik (,tokenism“) genutzt $\mathrm{zu}$ werden: ,they are hyper-visible, compared to their actual impact and effective participation, and this can be attributed to the large social media presence these initiatives have“" (Cardullo et al. 2018, S. 48). Neben den typischen Beteiligungsformen gibt es auch viele Arbeiten, die die Umsetzung der Beteiligung kritisieren und zum Beispiel die Beachtung von Dissens-Praktiken anstelle smarter Daten zur Identifikation von Brennthemen fordern (Kaika 2017) und dass die Partizipationsformen entsprechend der Forderungen von BürgerInnen angepasst werden (Przeybilovicz et al. 2020).

Hier kann e-Government dazu beitragen, das Augenmerk auf bereits verbreitet genutzte Technologien, wie Webseiten, Foren und soziale Medien zu lenken. Eine Besinnung auf die Grundsätze des kollaborativen Designs der IS hilft dagegen, eine menschenzentrierte technische Gestaltung zu verankern. In beiden Fällen sollte das Politische an der Auswahl von Artefakten beachtet werden und damit auch ihre sozio-technischen Auswirkungen.

\section{Fazit und Limitationen}

Die Analyse zeigt, dass es einige Smart-City-typische Bürgerbeteiligungsarten gibt, die eher im Bereich Information Systems Design zu finden sind als im Bereich e-Government. Dies betrifft besonders Living Labs und Mikrofabriken, die physische Orte der kollaborativen Arbeit an städtischen Problemen darstellen. Ein Schlüsselaspekt ist dabei Open Data, was als Grundlage für die Entwicklung von Artefakten genutzt werden kann oder Aufschluss über bestehende Probleme gibt. Datenplattformen werden durch Sensoren, aber auch die Einbindung von BürgerInnen durch Crowdsourcing und Citizen Sensing gespeist. Dabei gemeldete Missstände können 
wiederum als Grundlage für die Priorisierung städtischer Herausforderungen genutzt werden. Auch im Bereich Smart Governance wird eine bürgernahe, effiziente Erbringung staatlicher Dienstleistungen angestrebt. Dabei reicht das Spektrum vom bloßen Informieren (z.B. inklusive der Nutzung von Virtual-Reality-Anwendungen) bis zum Meinungsaustausch oder digital gestützte Abstimmungen. Dazu werden etablierte Kanäle wie soziale Medien oder speziell entwickelte Apps, Webseiten oder öffentliche Displays oder Terminals genutzt.

Die Analyse hat gezeigt, dass BürgerInnen zum Teil sowohl bei der technischen Entwicklung von Artefakten als auch bei der Ausrichtung der Stadtpolitik als IdeenträgerInnen, zur Abwägung von Priorisierungen als auch als TrägerInnen lokalen Wissens eingebunden werden. Ihr Input kann dabei sowohl durch Citizen Sensing oder Meldeplattformen eher technokratisch zur Verbesserung von Prozessen und Entwicklungen genutzt werden, oder transformativ zur Entscheidung über Priorisierungen und Gestaltung von Prozessen selbst. Ein guter Ausgangspunkt ist hierbei sowohl für technische als auch politische Prozesse der Appell nach ,empowering by design“ (Human et al. 2020, S. 4107). Auffällig ist, dass gerade auch Arten der Beteiligung, bei der BürgerInnen als Sensoren fungieren, weite Verbreitung finden in Smart Cities. Dabei ist zu beachten, dass es besonders dann nicht sinnvoll ist, BürgerInnen zu immer mehr Erfassung von Daten heranzuziehen, wenn diese in Silos verwahrt, monetarisiert oder nicht unter relevanten Behörden geteilt werden. Das Teilen von Daten wird besonders gefordert, um kreatives Potenzial zu unterstützen, sowie um eine polyzentrische Struktur zu ermöglichen (German Advisory Council on Global Change 2016). Gerade bei dem Zusammenführen und Teilen von Daten ist besonders zu beachten, dass diese nicht zur Überwachung von Individuen oder ausgewählten gesellschaftlichen Gruppen ausgenutzt werden - eine Kritik, der sich die Datenerfassung zur polizeilichen Strafverfolgung zum Teil gegenübersieht (Meijer und Wessels 2019). Technische Lösungen zur Wahrung der Privatsphäre besonders im Kontext von Smart Cities existieren bereits (MartinezBalleste et al. 2013), sie müssen allerdings konsequent implementiert werden.

Die hier dargestellten Ergebnisse sind limitiert durch die nicht-repräsentative Selektion der analysierten Beiträge, die allerdings ein weites Spektrum abdeckt, das typische Ausprägungen und lokale Anwendungsbeispiele verdeutlicht und damit zukünftige Projekte inspirieren kann. Als anschließende Arbeit wäre eine systematische Analyse von Smart-City-Anwendungsfällen vielversprechend, die die Häufigkeit und Charakteristika der jeweils genutzten technischen Werkzeuge der verschiedenen Beteiligungsarten darstellt, sowie deren Implementation anhand relevanter Dimensionen, insbesondere des Verhältnisses von bottom-up zu top-down Initiativen, deren Wandel während des Prozesses (Przeybilovicz et al. 2020), die thematische Auswahl, sowie die Anzahl, Inklusivität und Dauer der Einbindung (Macintosh 2004). Soziotechnische Analysen sollten hierbei dazu beitragen, Bedingungen für Partizipation zu identifizieren sowie technische Artefakte zu entwickeln, die eine inklusive und bottom-up geleitete Stadtpolitik unterstützen und BürgerInnen bedeutende Beteiligung, zum Beispiel als Freiwillige, ermöglichen - wobei Auswahl und Gestaltung von IT-Artefakten eine zentrale Rolle spielen (Heine et al. 2018; Kaufhold et al. 2020). Dabei sollten die genutzten Verfahren zur Beteiligung systematisch verfolgt und evaluiert werden. Als Beispiel für gute Praxis kann dabei der DIID Monitor 
Online-Partizipation dienen (DIID 2020), der einen systematischen Vergleich der in Nordrhein-Westfahlen genutzten Verfahren ermöglicht (Steinbach et al. 2020).

Bisherige Evaluationen deuten darauf hin, dass besonders die Erhöhung der Lebensqualität betrachtet werden sollte, die eher von den Erfolgen im Bereich Smart Living als von Beteiligungsformaten abzuhängen scheint (Bolívar und López-Quiles 2018). In der IS-Literatur wird daher besonders eine erfolgreiche Planung der Stadtentwicklung, unterstützt durch Information Systems, betont: „As good governance is closely related to planning with people, often described as decentralized planning, a public engagement with processes involved in the planning would be of great importance“ (Ismagilova et al. 2019, S. 20). Hier können besonders einige ambitionierte Smart-City-Initiativen als Vorbild dienen, die zum Beispiel technikgestützt Debatten um den öffentlichen Haushalt (,electronic participatory budgeting“") und damit die städtische Priorisierung unterstützen und dabei besonderes Augenmerk auf die Identifikation kontroverser Themen und Dissens legen (Cantador et al. 2020).

Danksagung Diese Forschungsarbeit wurde vom Bundesministerium für Bildung und Forschung (BMBF) und vom Hessischen Ministerium für Wissenschaft und Kunst (HMWK) im Rahmen ihrer gemeinsamen Förderung für das Nationale Forschungszentrum für angewandte Cybersicherheit ATHENE und durch die LOEWE Initiative des Landes Hessen im Rahmen des LOEWE Zentrums emergenCITY gefördert.

Funding Open Access funding enabled and organized by Projekt DEAL.

Open Access Dieser Artikel wird unter der Creative Commons Namensnennung 4.0 International Lizenz veröffentlicht, welche die Nutzung, Vervielfältigung, Bearbeitung, Verbreitung und Wiedergabe in jeglichem Medium und Format erlaubt, sofern Sie den/die ursprünglichen Autor(en) und die Quelle ordnungsgemäß nennen, einen Link zur Creative Commons Lizenz beifügen und angeben, ob Änderungen vorgenommen wurden.

Die in diesem Artikel enthaltenen Bilder und sonstiges Drittmaterial unterliegen ebenfalls der genannten Creative Commons Lizenz, sofern sich aus der Abbildungslegende nichts anderes ergibt. Sofern das betreffende Material nicht unter der genannten Creative Commons Lizenz steht und die betreffende Handlung nicht nach gesetzlichen Vorschriften erlaubt ist, ist für die oben aufgeführten Weiterverwendungen des Materials die Einwilligung des jeweiligen Rechteinhabers einzuholen.

Weitere Details zur Lizenz entnehmen Sie bitte der Lizenzinformation auf http://creativecommons.org/ licenses/by/4.0/deed.de.

\section{Literatur}

Alawadhi S, Aldama-Nalda A, Chourabi H et al (2012) Building understanding of smart city initiatives. In: International conference on electronic government. Springer, Berlin Heidelberg, S 40-53 https:// doi.org/10.1007/978-3-642-33489-4_4

Albino V, Berardi U, Dangelico RM (2015) Smart cities: definitions, dimensions, performance, and initiatives. J Urban Technol 22:3-21

Angelidou M (2015) Smart cities: a conjuncture of four forces. Cities 47:95-106. https://doi.org/10.1016/ j.cities.2015.05.004

Angelidou M (2016) Four European smart city strategies. Int J Soc Sci Stud. https://doi.org/10.11114/ijsss. v4i4.1364

Angelidou M (2017) The role of smart city characteristics in the plans of fifteen cities. J Urban Technol 24:3-28. https://doi.org/10.1080/10630732.2017.1348880

Araujo V, Mitra K, Saguna S, Åhlund C (2019) Performance evaluation of FIWARE: a cloud-based IoT platform for smart cities. J Parallel Distrib Comput 132:250-261. https://doi.org/10.1016/j.jpdc.2018. 12.010 
Arnstein SR (1969) A ladder of citizen participation. Journal of the American Institute of planners, 35(4):216-224

Bakici T, Almirall E, Wareham J (2013) A smart city initiative: the case of Barcelona. J Knowl Econ 4:135-148. https://doi.org/10.1007/s13132-012-0084-9

Bencardino M, Greco I (2014) Smart communities. Social innovation at the service of the smart cities. TeMA J L Use Mobil Environ. https://doi.org/10.6092/1970-9870/2533

Benevolo C, Dameri RP, D’Auria B (2016) Smart mobility in smart city. In: Torre T, Braccini A, Spinelli R (Hrsg) Empowering organizations, 11. Aufl. Lecture Notes in Information Systems and Organisation. Springer, Cham https://doi.org/10.1007/978-3-319-23784-8_2

Benouaret K, Valliyur-Ramalingam R, Charoy F (2013) CrowdSC: building smart cities with large-scale citizen participation. IEEE Internet Comput 17:57-63. https://doi.org/10.1109/MIC.2013.88

Billert MS, Peters C (2019) Die Digitalisierungsstraße für die Stadt der Zukunft - Kollaborative Entwicklung eines Portals für bürger-initiierte Dienstleistungsentwicklung im Kontext einer Smart City. HMD 56:172-189. https://doi.org/10.1365/s40702-018-00490-6

Bitkom (2019) Smart-City-Atlas. Die kommunale digitale Transformation in Deutschland, S 1-194

Boes K, Buhalis D, Inversini A (2015) Conceptualising smart tourism destination dimensions. In: Tussyadiah I, Inversini A (Hrsg) Information and communication technologies in tourism 2015. Springer, Cham https://doi.org/10.1007/978-3-319-14343-9_29

Bolívar MPR, López-Quiles JM (2018) The quest for the quality of life in european smart cities. An empirical research. 19th Annu Int Conf Digit Gov Res dg.o'18. https://doi.org/10.1145/3209281. 3209296

Brown CC (2017) Google scholar. Charlest Advis 19:31-34

Cantador I, Cortés-Cediel ME, Fernández M (2020) Exploiting open data to analyze discussion and controversy in online citizen participation. Inf Process Manag. https://doi.org/10.1016/j.ipm.2020.102301

Capdevila I, Zarlenga MI (2015) Smart city or smart citizens? The Barcelona case. J Strateg Manag 8:266-282. https://doi.org/10.1108/JSMA-03-2015-0030

Cardone G, Foschini L, Bellavista P et al (2013) Fostering participaction in smart cities: a geo-social crowdsensing platform. IEEE Commun Mag 51:112-119. https://doi.org/10.1109/MCOM.2013. 6525603

Cardullo P, Kitchin R (2019) Being a 'citizen' in the smart city: up and down the scaffold of smart citizen participation in Dublin, Ireland. GeoJournal 84:1-13. https://doi.org/10.1007/s10708-018-9845-8

Cardullo P, Kitchin R, Di Feliciantonio C (2018) Living labs and vacancy in the neoliberal city. Cities 73:44-50. https://doi.org/10.1016/j.cities.2017.10.008

Castelnovo W, Misuraca G, Savoldelli A (2016) Smart cities governance: the need for a holistic approach to assessing urban participatory policy making. Soc Sci Comput Rev 34:724-739. https://doi.org/10. $1177 / 0894439315611103$

Chang H-Y (2019) Let's talk about Smart City! Engaging citizens with Smart City through dialogues evoked by playfuland speculative experience design. TU, Delft

Chatfield AT, Reddick CG (2016) Smart city implementation through shared vision of social innovation for environmental sustainability: a case study of Kitakyushu, Japan. Soc Sci Comput Rev 34:757-773. https://doi.org/10.1177/0894439315611085

Cohen B (2014) The smartest cities in the world 2015: methodology. https://www.fastcompany.com/ 3038818/the-smartest-cities-in-the-world-2015-methodology. Zugegriffen: 16. Juli 2021

Degbelo A, Granell C, Trilles S et al (2016) Opening up smart cities: citizen-centric challenges and opportunities from GIScience. ISPRS Int J Geo-information. https://doi.org/10.3390/ijgi5020016

Development $\mathrm{O}$ for EC and (2001) Citizens as partners: OECD handbook on information, consultation and public participation in policy-making. OECD Publishing,

DIID (2020) Monitor Online-Partizipation. https://www.monitor-online-partizipation.de/informationenzum-projekt. Zugegriffen: 16. Juli 2021

Effing R, Groot BP (2016) Social smart city: Introducing digital and social strategies for participatory governance in smart cities. In: 5th International Conference on Electronic Government and Information Systems Perspective (EGOV), S 241-252

Farías I, Widmer S (2017) Ordinary Smart Cities: how calculated users, professional citizens, technology companies and city administrations engage in a more-than-digital politics. Ital J Sci Technol Stud 8:43-60

Gabrys J (2014) Programming environments: environmentality and citizen sensing in the smart city. Environ Plan D Soc 32:30-48. https://doi.org/10.1068/d16812 
German Advisory Council on Global Change (WBGU) (2016) Humanity on the move: Unlocking the transformative power of cities. Flagship Report. Flagsh. https://issuu.com/wbgu/docs/hg2016_en_ highres? $=37591641 / 68733616$. Zugegriffen: 2 . August 2021

Giatsoglou M, Chatzakou D, Gkatziaki V et al (2016) Citypulse: a platform prototype for smart city social data mining. J Knowl Econ 7:344-372. https://doi.org/10.1007/s13132-016-0370-z

Giffinger R, Fertner C, Kramar H, Meijers E (2007) City-ranking of European medium-sized cities. Cent Reg Sci Vienna UT, S 1-26

Gooch D, Wolff A, Kortuem G, Brown R (2015) Reimagining the role of citizens in smart city projects. Proc 2015 ACM Int Jt Conf Pervasive Ubiquitous Comput, S 1587-1594 https://doi.org/10.1145/ 2800835.2801622

Goodchild MF (2007) Citizens as sensors: the world of volunteered geography. Geoj 69:211-221. https:// doi.org/10.1007/s10708-007-9111-y

Granier B, Kudo H (2016) How are citizens involved in smart cities? Analysing citizen participation in Japanese "smart Communities.". Inf Polity 21:61-76. https://doi.org/10.3233/IP-150367

Heine M, Kuper S, Neururer T (2018) Which platform to use? Social media platform types and their suitability for sound decision making by voluntary helpers. ICEGOV'18, S 395-402 https://doi.org/ $10.1145 / 3209415.3209485$

Hivon J, Titah R (2017) Conceptualizing citizen participation in open data use at the city level. Transform Gov People Proc Policy 11:99-118. https://doi.org/10.1108/TG-12-2015-0053

Hosio S, Goncalves J, Kukka H (2014) Situated engagement and virtual services in a smart city. In: IEEE 7th International Conference on Service-Oriented Computing and Applications SOCA 2014. https:// doi.org/10.1109/SOCA.2014.31

Hu X, Chu THS, Chan HCB, Leung VCM (2013) Vita: a crowdsensing-oriented mobile cyber-physical system. IEEE Trans Emerg Top Comput 1:148-165. https://doi.org/10.1109/TETC.2013.2273359

Human S, Gsenger R, Neumann G (2020) End-user empowerment: an interdisciplinary perspective. In: 53rd Hawaii International Conference on System Sciences, S 4102-4111

Ismagilova E, Hughes L, Dwivedi YK, Raman KR (2019) Smart cities: advances in research-An information systems perspective. Int J Inf Manage 47:88-100. https://doi.org/10.1016/j.ijinfomgt.2019. 01.004

Jussila J, Kukkamäki J, Mäntyneva M, Heinisuo J (2019) Open data and open source enabling smart city development: a case study in Häme region. Technol Innov Manag Rev 9:25-34. https://doi.org/10. 22215/timreview/1266

Kaika M (2017) 'Don’t call me resilient again!': the New Urban Agenda as immunology ... or ... what happens when communities refuse to be vaccinated with 'smart cities' and indicators. environ urban 29:89-102. https://doi.org/10.1177/0956247816684763

Kaufhold M-A, Haunschild J, Reuter C (2020) Warning the public: a survey on attitudes, expectations and use of mobile crisis apps in Germany. In: Proc Eur Conf Inf Syst, S 1-16

Kitchin R (2014) The real-time city? Big data and smart urbanism. GeoJournal 79:1-14. https://doi.org/ $10.1007 / \mathrm{s} 10708-013-9516-8$

Kitchin R (2015) Making sense of smart cities: addressing present shortcomings. Cambridge J Reg Econ Soc 8:131-136. https://doi.org/10.1093/cjres/rsu027

Komninos N, Pallot M, Schaffers H (2013) Special issue on smart cities and the future Internet in Europe. J Knowl Econ. https://doi.org/10.1007/s13132-012-0083-x

Kummitha RKR, Crutzen N (2017) How do we understand smart cities? An evolutionary perspective. Cities 67:43-52. https://doi.org/10.1016/j.cities.2017.04.010

Lawton P, Murphy E, Redmond D (2014) Neoliberalising the city 'creative-class' style. In: Neoliberal urban policy and the transformation of the city. Springer, Berlin Heidelberg, S 189-202

Lebrument N, Zumbo-Lebrument C, Rochette C, Roulet TJ (2021) Triggering participation in smart cities: political efficacy, public administration satisfaction and sense of belonging as drivers of citizens' intention. Technol Forecast Soc Change 171:120938. https://doi.org/10.1016/j.techfore.2021.120938

Lytras MD, Visvizi A, Sarirete A (2019) Clustering smart city services: Perceptions, expectations, responses. Sustainability 11:1-19. https://doi.org/10.3390/su11061669

Macintosh A (2004) Characterizing E-participation in policy-making. In: Proceedings of the 37th Hawaii international conference on system sciences, S 1-10

Mainka A, Castelnovo W, Miettinen V et al (2016) Open innovation in smart cities: civic participation and co-creation of public services. Proc Assoc Info Sci Tech 53:1-5. https://doi.org/10.1002/pra2.2016. 14505301006 
Manville C, Cochrane G, Cave J et al (2014) Mapping Smart Cities in the EU. European Parliament. https://www.europarl.europa.eu/RegData/etudes/etudes/join/2014/507480/IPOL-ITRE_ET\%282014 \%29507480_EN.pdf. Zugegriffen: 2. August 2021

Martinez-Balleste A, Perez-Martinez P, Solanas A (2013) The pursuit of citizens' privacy: a privacy-aware smart city is possible. IEEE Commun Mag 51:136-141. https://doi.org/10.1109/MCOM.2013. 6525606

Meijer A, Bolívar MPR (2016) Governing the smart city: a review of the literature on smart urban governance. Int Rev Adm Sci 82:392-408. https://doi.org/10.1177/0020852314564308

Meijer A, Thaens M (2018) Urban technological innovation: developing and testing a sociotechnical framework for studying smart city projects. Urban Aff Rev. https://doi.org/10.1177/1078087416670274

Meijer A, Wessels M (2019) Predictive policing: review of benefits and drawbacks. Int J Public Adm 42:1031-1039. https://doi.org/10.1080/01900692.2019.1575664

Meijer A, Lips M, Chen K (2019) Open governance: a new paradigm for understanding urban governance in an information age. Front Sustain Cities. https://doi.org/10.3389/frsc.2019.00003

Mellouli S, Luna-Reyes LF, Zhang J (2014) Smart government, citizen participation and open data. Inf Polity 19:1-4

Menny M, Voytenko Palgan Y, McCormick K (2018) Urban living labs and the role of users in co-creation. GAIA. https://doi.org/10.14512/gaia.27.S1.14

Nam T, Pardo TA (2011) Conceptualizing smart city with dimensions of technology, people, and institutions. In: 12th annual Internat. Conference on digital government research, S 282-291

Niaros V (2016) Introducing a taxonomy of the "smart city": Towards a commons-oriented approach. TripleC 14:51-61. https://doi.org/10.31269/triplec.v14i1.718

Okai E, Feng X, Sant P (2019) Smart cities survey. Proc-20th Int Conf High Perform Comput Commun 16th Int Conf Smart City 4th Int Conf Data Sci Syst, S 1726-1730 https://doi.org/10.1109/HPCC/ SmartCity/DSS.2018.00282

Oksman V, Raunio M (2018) Citizen-centric smart city planning for africa: a qualitative case study of early stage co-creation of a namibian smart community. In: The twelfth international conference on digital society and egovernments, S 30-35

Oliveira Á, Campolargo M (2015) From smart cities to human smart cities. In: Proceedings of the annual Hawaii international conference on system science

Pellicano M, Calabrese M, Loia F, Maione G (2019) Value co-creation practices in smart city ecosystem. J Serv Sci Manag 12:34-57. https://doi.org/10.4236/jssm.2019.121003

Petrin J (2012) nexthamburg. Bürgervision für eine neue Stadt, Edition Körber-Stiftung. https://issuu.com/ nexthamburg/docs/nexthamburg_buergervision_online. Zugegriffen: 2. August 2021

Portmann E, Finger M (2015) Smart Cities - Ein Überblick! HMD 52:470-481. https://doi.org/10.1365/ s40702-015-0150-4

Przeybilovicz E, Cunha MA, Geertman S et al (2020) Citizen participation in the smart city: findings from an international comparative study. Local Gov Stud. https://doi.org/10.1080/03003930.2020.1851204

Reforgiato Recupero D, Castronovo M, Consoli S et al (2016) An innovative, open, Interoperable citizen engagement cloud platform for smart government and users' interaction. J Knowl Econ 7:388-412. https://doi.org/10.1007/s13132-016-0361-0

Reuter C, Haunschild J, Hollick M et al (2020) Towards secure urban infrastructures: cyber security challenges to information and communication technology in smart cities. In: Mensch und Computer 2020 - Workshopband. Gesellschaft für Informatik e. V., Magdeburg

Sadowski J, Bendor R (2019) Selling smartness: corporate narratives and the smart city as a sociotechnical imaginary. Sci Technol Hum Values 44:540-563. https://doi.org/10.1177/0162243918806061

Schuurman D, Baccarne B, De Marez L, Mechant P (2012) Smart ideas for smart cities: Investigating crowdsourcing for generating and selecting ideas for ICT innovation in a city context. J Theor Appl Electron Commer Res 7:49-62. https://doi.org/10.4067/S0718-18762012000300006

Shelton T, Zook M, Wiig A (2015) The "actually existing smart city. Cambridge J Reg Econ Soc 8:13-25. https://doi.org/10.1093/cjres/rsu026

Simonofski A, Hertoghe E, Steegmans M et al (2021) Engaging citizens in the smart city through participation platforms: a framework for public servants and developers. Comput Human Behav 124:106901. https://doi.org/10.1016/j.chb.2021.106901

Simonofski A, Asensio ES, De Smedt J, Snoeck M (2019) Hearing the voice of citizens in smart city design: the citivoice framework. Bus Inf Syst Eng 61:665-678. https://doi.org/10.1007/s12599-0180547-z

Sørensen E, Torfing J (2018) Co-initiation of collaborative innovation in urban spaces https://doi.org/10. $1177 / 1078087416651936$ 
Spagnoli F, van der Graaf S, Brynskov M (2019) The paradigm shift of living labs in service co-creation for smart cities: synchronicity validation. In: Lazazzara A, Nacamulli R, Rossignoli C, Za S (Hrsg) Organizing for digital innovation, 27. Aufl. LNISO. Springer, Berlin Heidelberg, S 135-147 https:// doi.org/10.1007/978-3-319-90500-6_11

Spil TAM, Effing R, Kwast J (2017) Smart city participation: dream or reality? A comparison of participatory strategies from hamburg, Berlin \& Enschede. In: 16th IFIP Conference on e-Business, e-Services, and e-Society I3E 2017. Springer, Berlin Heidelberg, S 122-134 https://doi.org/10.1007/ 978-3-319-68557-1_12

Steinbach M, Wilker N, Schöttle S (2020) E-participation on the local level-a census survey approach for researching its implementation. J Inf Technol Polit 17:12-32. https://doi.org/10.1080/19331681. 2019.1676361

Strömberg F, Näslund E (2017) Open data within a smart city initiative: a case study exploring how collaboration can foster innovation within a smart city initiative. Dissertation

Vanolo A (2014) Smartmentality: the smart city as disciplinary strategy. Urban Stud 51:883-898. https:// doi.org/10.1177/0042098013494427

Viale Pereira G, Cunha MA, Lampoltshammer TJ et al (2017) Increasing collaboration and participation in smart city governance: a cross-case analysis of smart city initiatives. Inf Technol Dev 23:526-553. https://doi.org/10.1080/02681102.2017.1353946

Waze (2020) About us. www.waze.com/about. Zugegriffen: 16. Juli 2021

Weed M (2005) "Meta interpretation" A method for the interpretive synthesis of qualitative research. Forum Qual Sozialforsch. https://doi.org/10.17169/fqs-6.1.508

Wiig A (2016) The empty rhetoric of the smart city: from digital inclusion to economic promotion in Philadelphia. Urban Geogr 37:535-553. https://doi.org/10.1080/02723638.2015.1065686

Wilson K, Cohen S, Mar C et al (2013) Microsoft citynext-technical reference. http://download. microsoft.com/download/8/C/D/8CD464C6-44BA-45E9-B104-32014E99A51F/Microsoft\%20 CityNext\%20Technical\%20Reference\%20Model\%20Overview.pdf. Zugegriffen: 16. Juli 2021

$\mathrm{Xu} \mathrm{H}$, Zhu W (2020) Evaluating the impact mechanism of citizen participation on citizen satisfaction in a smart city. Environ Plan B Urban Anal City Sci. https://doi.org/10.1177/2399808320980746

Yeh H (2017) The effects of successful ICT-based smart city services: from citizens' perspectives. Gov Inf Q 34:556-565. https://doi.org/10.1016/j.giq.2017.05.001

Zandbergen D (2017) "We are Sensemakers": the (anti-)politics of smart city co-creation. Public Cult 29:539-562. https://doi.org/10.1215/08992363-3869596

Zanella A, Bui N, Castellani A et al (2014) Internet of things for smart cities. IEEE Internet Things J 1:22-32. https://doi.org/10.1109/JIOT.2014.2306328 